\title{
Road Signs Perception Evaluation by Means of a Semi-immersive Tool
}

\author{
Roberto Vigano, Edoardo Rovida, Riccardo Vincenti and Marco Ramondino
}

Politecnico di Milano - Dipartimento di Meccanica , Italy

\begin{abstract}
To reduce the number of road accident victims the European Commission has encouraged the European member states to implement a series of actions in this field. These actions include the development of intelligent and integrated safety systems as well as educational and training initiatives.

Educational initiatives include the training of the drivers to improve their ability and sense of responsibility. In addition to the direct use of the vehicle, the training includes the recognition of the traffic signs. Since the recognition may be influenced by both the position of the signal and the weather conditions, the authors have studied the possibility of evaluate the drivers' perception of road signs by means of a virtual environment tool able to perform different operative conditions. A series of tests was conducted to evaluate the visualization tool created and its ability to replace other recognition tests. This paper reports first tests results.
\end{abstract}

Index Terms - Comprehension, Perception, Traffic signs, Simulator.

\section{INTRODUCTION}

In 1997 the European Community created the "Second programme of action" plan with the objective of reduce the number of road accident victims of the 40\% within 2010. Subsequently, in 2001, the target was moved to $50 \%$ as explained in the "White book on Transports European policy for 2010: Time to Decide" plan [1]. This last plan estimates about 45 billions of euro per year the cost to sustain road accidents in Europe and multiplies it by four including psychological damages to people and others indirect road accidents. Continuing along this path, in 2002, the European Commission formed the eSafety working group to encourage the development and use of intelligent and integrated safety systems in vehicles. A statistics reported on the web site of eSafety group [2] estimates that about 1.2 million of people in Europe were injured in road accidents in 2005, and over 40,000 killed. An important consideration that can be deducted from analysis of accidents is that over 93 per cent of them resulted from human error.

Following the indications of the European Commission, in the last years, a number of plans to improve road safety in most of European member states has been implemented. Italy, for example, has planned and realized a series of actions in the road transport field. These actions include: educational

Manuscript received on February 7, 2011

Email: roberto.vigano@polimi.it and regulatory initiatives, like compulsory driver licence for mopeds and motorcycles and mandatory use of helmets; sanctions, like driver licence on penalty point; safety measures on the infrastructures. These Italian initiatives have led to a reduction of the number of road accident victims in the period between 2001 and 2005 of the 5\%, and an average decrease of the $4 \%$ per year of the number of accidents, against a reduction of the mortality caused by road accidents only of $2 \%$ per year between 1994 and 2000, and an increase of the number of collision. Despite these data, the "European Road Statistic 2008" [3] report, that summarize the situation in Europe in 2006, evidences like the results obtained are not enough and that the actions taken must still improve their effectiveness.

If it is unquestioned that the actions taken have been able to reduce the number and the seriousness of accidents, it is necessary to remember that these benefits have involved principally the drivers of vehicles with four wheels and only marginally those of two-wheeled motorized vehicles. The latter are increasing according to a rate of growth from which one can assume that at the end of the 2010, the percentage of road accident victims of the two wheels will double.

The reason for the higher mortality of motorcycle riders is certainly the low protection offered in case of accident or falls, both by vehicles and by the protections provided by the clothing of the rider. Often, the accident was caused by unexpected changes in road conditions or because of the presence of unexpected obstacles, like animals or objects on the road. Although in some cases the accidents are attributable to bad luck, in many cases they have been caused by negligence of the rider, such as the ignorance of the actual performance of the motorcycle or the lack of knowledge of the rules of the road Code.

The trend of the statistical data regarding motorcycles is imputable to the increment of the transport on two wheels, especially in the city environment. The increment of the motorcycles on the road is due to the small size of the vehicles that allows to fit easily in tight spaces, permitting a good level of flexibility to the users in congested traffic. Thus, further attention should be putting to these new road users, so as to increase their ability to identify hazards while driving [4].

The authors think that aid in these cases might come from the study of tools that improve the skill of riders and their sense of responsibility while driving. Following this idea, the research undertaken at the Mechanical Department of the Politecnico di Milano aims to face the problem through the development of a training simulator able to immerse the user in a realistic driving experience, such as regards both traffic and weather conditions. It is believed that the training of users of two-wheeled vehicles 
to the traffic situations and of danger by means of virtual tools, may be a way for aid them to drive with more caution and to improve their sense of what will happen. This training method should be, also, integrated with the practice currently adopted for the acquisition of driver's license. The ambition of the work is therefore to create a virtual tool able to support the learning process, not just understanding the rules governing road traffic but, also, to teach to the future users of two-wheeled vehicles, the respect of these rules. The use of a simulation system offers a high degree of experimental control, minimizing the safety issues associated to the driving procedures on road and on test-track. Also, the simulator permits to realize experiences that may be extremely both expensive and time consuming in a real-world environment.

The research activity devoted to the development of the simulator system was divided into three main functional parts, namely:

- Visual environment

- Ergonomics and human interaction

- Vehicle frame and motion

The subdivision was realized in order to better analyze the issues connected to the implementation both of the hardware and the software of the simulator system.

This paper is focused on this first activity of the research project, about the definition of the visual environment of the simulator, and explains the experience realized and the results obtained.

To evaluate the performance and the implementation of the visual stimuli required to the simulator system, a series of tests were conducted on different solutions. These tests were devoted to evaluate the user' capacity to identify some traffic signals correctly during a simulation stage using a monitor and a Head Mounted Display (HMD). The different solutions analysed have involved the changing of the weather conditions and the presence of road vehicles on the path.

\section{STATE OF THE ART}

Since the year ' 60 s, in many countries the need to develop simulators to improve driving conditions and to reduce traffic accidents was felt, but only from ' 80 s the automobile manufacturers began to develop their own driving simulators [5]. Initially, the simulators were designed to replicate the dynamics of the vehicles, with complicated mechanisms requiring expensive control systems, and only after, the designers were focused on the ergonomic studies and human interactions. Such as for the four-wheeled vehicles simulators, the motorcycle simulators were build, in the last years, with the purpose of studying the motorcycle dynamic and maneuverability, as well as the man-vehicle interaction.

The first simulator was developed by Honda in 1988, with the purpose of studying the motorcycle stability and its maneuverability [6]. After 1990, Honda launches a second prototype, more simple that the first, to permit tests in safe conditions, followed by a third prototype developed using a parallel manipulator with six degree of freedom (DOF), and with a HMD for visual projection [7]. In 1995 was developed, at the PERCRO laboratory, the MORIS simulator consisting of a real scooter mounted on a Stewart parallel platform with seven DOF [8]. Finally, a motorcycle simulator was developed at the University of Padova (Italy) in order to study the man-vehicle interaction [9]. All of these simulators have been initially developed to reproduce the dynamics behavior of the vehicle with the purpose to obtain information useful to the motorcycle design.

In January 2006 Honda Co., Ltd announces marketing of his riding simulator for training [10], evolution of the simulator already developed ten years earlier. Its aim was to teach, to future users of two-wheeled vehicles, the road traffic rules and to both estimate and prevent danger situations simulating real traffic conditions. The simulator consists in a static structure that receives the rider, forcing a posture similar to that found on a scooter. The visualization system is composed by a monitor installed in front to the head of the user. It also includes an audio system to reproduce the traffic noise. The user can control the vehicle direction applying a torque on the handlebars and adjust the speed by means of the clutch and gear commands. A similar solution was presented by Mitsubishi Co., Ltd. in cooperation with SUZUKI Motor Corp. and YAMAHA MOTOR Co., Ltd. In this case the simulator permits the selection of the performances of the virtual vehicle, from 50 cc. to 400 cc. The structure that receive the user and reproduce the posture of the two wheeled vehicle can be either a real motorcycle or a mock-up.

Many initiatives to raise awareness on the driving behaviours of two- and four-wheeled vehicles have emerged in recent years in European countries. On this wave, many companies have developed systems of training specifically targeted to young people who wish to obtain a driver's license for motorcycles. In Italy, for example, Koala Games s.r.l., specialized in the creation of training videogames, has developed the GUIDATU PRO project [11]. This project is devoted to reproduce both the driving position and the control commands of vehicles. This simulator is proposed to the driving schools to support the learning of the road safety education. It is able to simulate the infringements of the road code and the related penalties. The visualization system is composed by a monitor, able to reproduce the 3D environment, installed in front of the user.

It should be noted that simulators are considered tools useful and reliable to assess the driver's behaviour caused by the traffic conditions. The driving simulators are also useful to the analysis of the visual perception process that the driver uses during the guidance [12]. This permits the use of the driving simulators to the assessment, both of the understanding capacity of the traffic signals by the users and of the quality of the road signs including the position on the road. The traffic signals are a form of universal language, thanks to some typical characteristics that make them understandable in the world. The traffic signals are governed by rules, concerning adopted symbols, materials and position on the road, that determine their visibility and legibility both day and night. Unfortunately the implementation of these rules is often not met, and the traffic signals are often absent or barely visible, especially in adverse weather conditions and at night. This could be cause for road accidents. In fact, studies conducted by INRETS (The 
French National Institute for Transport and Safety Research) [13] and those derived from the survey sample called REAGIR show that $10 \%$ of respondents indicate the traffic signals as the cause of the accidents; a proportion comparable with those arising from factors such as the characteristics of the road.

One other cause of road accidents is, however, the recognition lack of the traffic signal by the driver. This is often due to the high speed of the vehicle, which reduces the time available for signal recognition. Many methods exist for testing the traffic signal comprehension. Ideally, the design of the new traffic signal may be tested in simulation or on the road. Chrysler et al. [14] noted that, conceptually, performing the correct manoeuvre and at the appropriate time after viewing a traffic signal, a driver could indicate the correct comprehension of the rule imposed by the signal. The same report underline that the recognition of traffic signs is affected by the viewing time. The classical evaluation tests, based on pen and pencil, seem to provide results of the comprehension of traffic signals most optimistic than the tests that leave little time for the recognition of the signal. Many tests abaut the recognition and the comprehension of the traffic signals are conducted using monitors or large projection screens that are more suitable for the study of the driving on cars. This configuration of the display is less suitable for riders of motorcycles, where the presence the helmet can affect their capacity and breadth of vision.

On basic of these considerations, the study was directed to assessing the ability of perception and recognition of traffic signals by means of a monitor or through a Head Mounted Display.

\section{REALIZATION OF THE TEST ENVIRONMENT}

When you plan to replicate a real situation using a simulator is considered important to be able to provide "sensations" to the user closer to the real ones. Where, with the term "sensations" we intend the man-vehicle interactions that derive from the forces of inertia acting on the rider while in motion, the dynamic responses both from the vehicle and from the road, the stimuli coming from the external environment (lights, sounds, weather, etc.).

We consider two types of use of driving simulators: a use-oriented to the study of human-vehicle interaction and one devoted to training. In the first case, the purpose of simulation is to obtain appropriate information to improve the behaviour of the vehicle and its ergonomics. This requires a simulator that can replicate as closely as possible the dynamics and the environment of the driving. In the second case, the main purpose is to stimulate the user to properly carry out some actions, measuring the reaction times and the behaviour. In the latter case, the simulator can be equipped with a dynamics less refined.

Following this last statement and for the purpose that this study aims, it is not necessary a correct description of the vehicle dynamics, but it is fundamental a creation of the virtual environment able to describe as closely as possible the environment in which the driving simulation occurs, the climatic, lighting and traffic conditions. For this, in order to assess the ability to recognize traffic signals by users in different environmental conditions, the study has been focused on the creation of a virtual reality simulation oriented mainly to the visual stimuli.

To the development of the process for the simulation representation, the Virtools system from Dassault Systèmes (www.virtools.com) was used. This application is 3D authoring system for rapidly developing interactive and 3D content in real-time. In Virtools the content is visually created by linking logical components (building blocks) to a workflow. Input and output ports of these building blocks (BBs) are connected by links and they help to implement a data and control flow within a visual scripting environment (Fig. 1). A scripting language together with a Software Development Kit (SDK) allow to develop complex applications and custom extensions. The "Physical pack" of Virtools was used into the study to define the behaviour of the motorcycle on the road, while keyboard and joystick were used to devices of control.

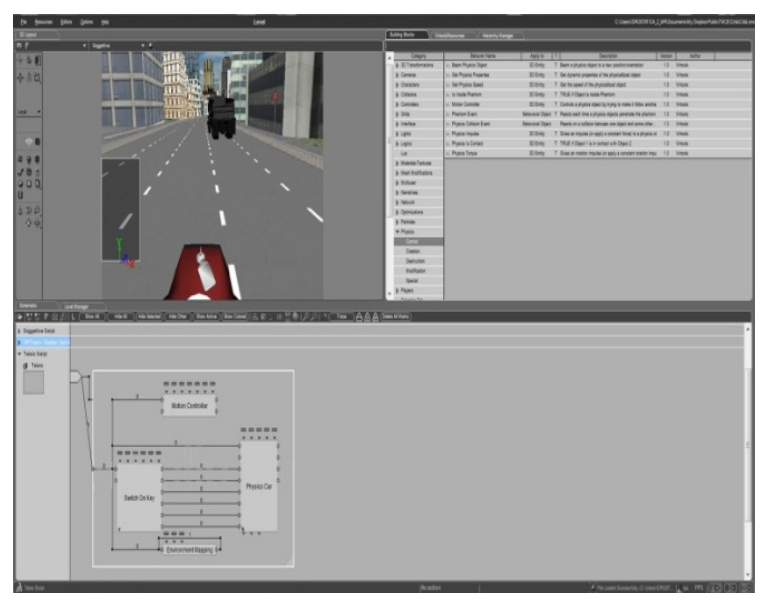

Fig. 1. Visual authoring system in Virtools.

In order to evaluate different conditions of driving, two simulation environments were created: a rural and an urban environment. The urban setting is characterised by a higher density of intersections, by the introduction of traffic lights and of elements of street furniture (Fig. 2). The rural environment scenario, instead, was reproduced by a road characterized by the presence of several trees that make annoying, in some cases, the proper visibility of the traffic signals, while, in other cases, they help the perception of the path development of the road (Fig. 3).

A further differentiation of simulation scenarios was achieved by introducing different weather situations. So, for each environment have been realised, by means of scripts, various climatic conditions, able to modify the visibility level of the scenario itself. In particular, three weather conditions were defined: a dry condition (sun), a wet condition (rain) and a low visibility condition (fog). In the Figures 3, 4 and 5 it is possible to appreciate the difference of the visualization depth in the three conditions.

In implementing the model, the motorcycle components and their relative degrees of freedom were identified. So, the following breakdown of the vehicle was introduced: 


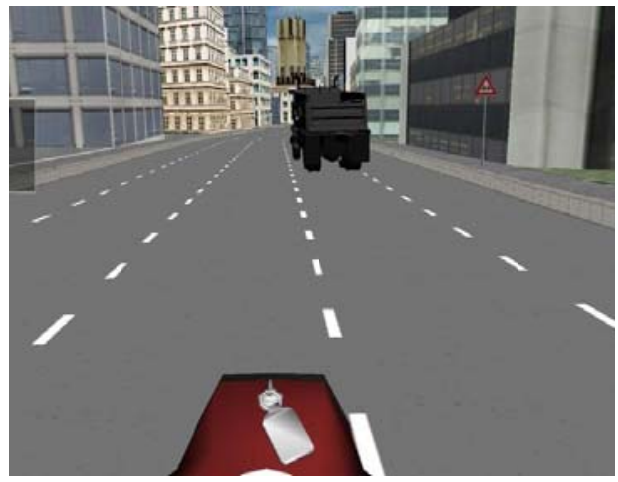

Fig. 2. Example of urban environment visualization.

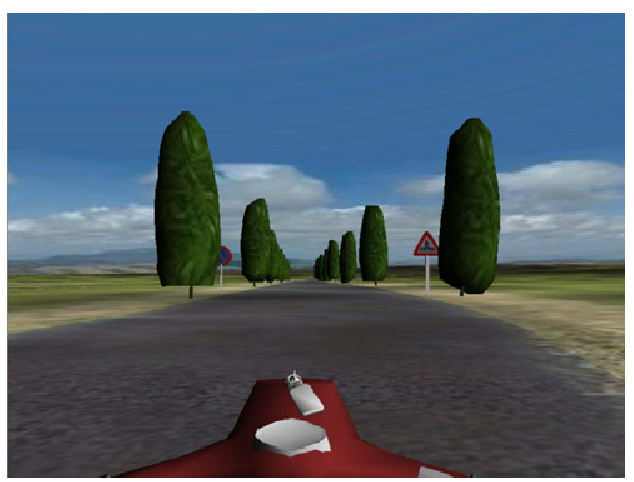

Fig. 3. Example of rural environment visualization in dry conditions.( Color Plate 5)

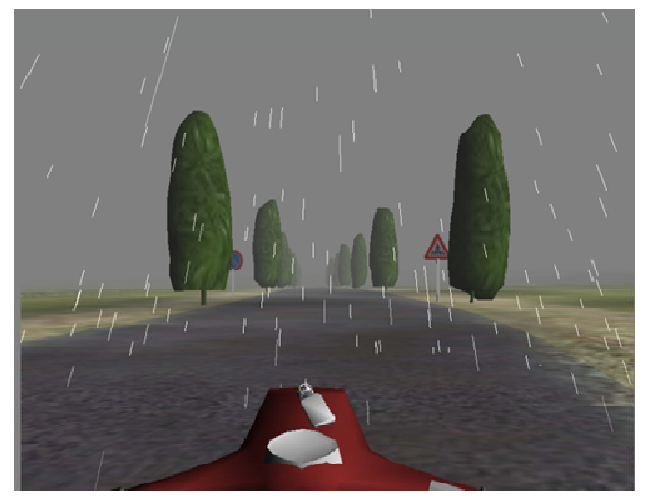

Fig. 4. Example of rural environment in wet conditions.

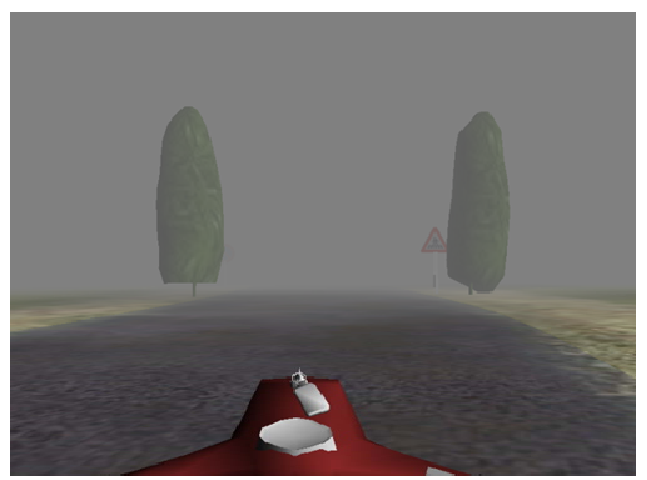

Fig. 5. Example of rural environment in low visibility conditions.

- The frame;
- The fork and the handlebars;

- The swing arm;

- Front wheel;

- Rear wheel.

With this scheme of the elements that make up the vehicle, it was possible to consider the movement imposed by the road irregularities; moreover, in order to have a more realistic simulation, it was also permitted the roll movement to the model. The physical behaviour model of the motorcycle was realized by means of a "building block" already existing in the software used to develop the simulation environment. The pitch movement and the roll movement of the vehicle have been defined by means of appropriate scripts, able to take in account the specific conditions of stability of the motorcycle.

It was, also, necessary to conduct a calibration of the model to make more realistic the behaviour of the vehicle into the range of the speeds forecasted to the simulation. In particular, some parameters was introduced with refer to the mass and inertial moments of the vehicle, the damping and the stiffness of the suspensions, the friction value, the gear ratio and the power of the engine. The limit speed was set to $50 \mathrm{~km} / \mathrm{h}$ in urban roads and to $90 \mathrm{~km} / \mathrm{h}$ in the rural environment. Basing on these speeds, the values of the physical parameters of the vehicle were defined and assigned as those of a typical scooter (Fig.6).

To reproduce the point of view of the rider it was introduced a camera positioned at the position of the user's eyes. In relation to the purpose of the test, were considered two control conditions of the orientation of the head of the pilot: one fixed and one mobile. In the case of on-screen display has not been considered any chance of head orientation. Therefore, the viewing position was placed at a certain height with respect to the vehicle and it was kept fixed. In the case of viewing through HMD were also considered the three rotations of the user's head: left-right and up-down and the roll movement around the longitudinal axis, always keeping the centre of gravity of the driver's head in a fixed position with respect to the vehicle. In both cases, the camera view is linked to the motion resulting from vehicle dynamics, assuming the pilot rigidly attached to the motorcycle.

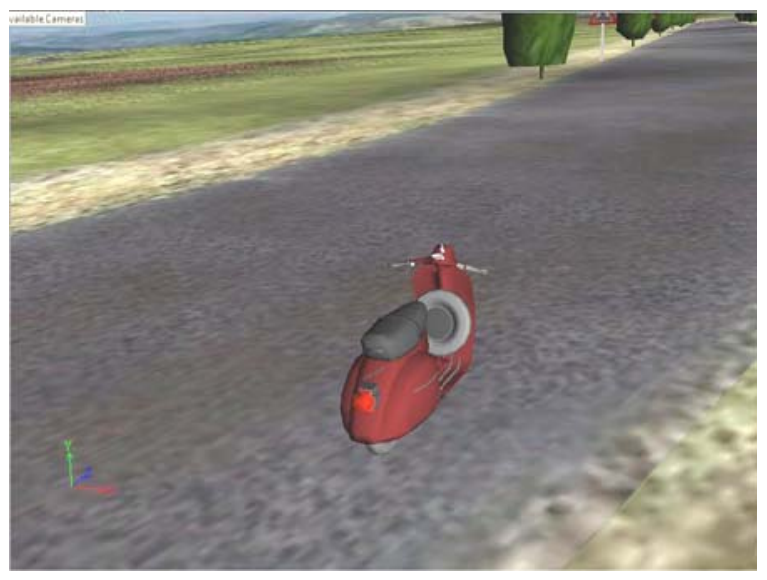

Fig. 6. The mock-up of the motorcycle used in simulation.( Color Plate 6 ) 
The possibility of introducing the three degrees of rotational freedom for the driver's head is due to the fact that the position of the HMD system is traced through a system of infrared cameras (VICON motion system). So it was possible to know the position and orientation of the head of the pilot at any time of the simulation. During the study, however, the position of the centre of gravity of the user's head has been neglected, taking account only of its orientation.

The objective has been to create a more realistic simulation also considering the movements of the head, that are fundamental to have an appropriate field of vision. Therefore, the user is able to explore the surroundings to catch as possible as visual information. To increase the realism of the simulation, in the case of vision through HMD, was also used stereoscopic vision.

To the control of the vehicle have been implemented the channels of connection with the BBs of the software necessary to manage the rotation of the handlebars and both acceleration and braking systems. Three channels were also established for the management of different climatic conditions, so that you can easily go from dry to wet and to fog. Finally, other three channels were activated to control the user interaction needed to manage the responses of the test.

All these channels have been linked to some keys on the keyboard. In particular, the arrow keys are used to manage the movement of the vehicle, while the keys $\mathrm{Z}, \mathrm{X}$ and $\mathrm{C}$ were used for the answers to the tests.

\section{THE SIMULATION PROTOCOL}

The present work was devoted to evaluate the user' capacity to identify some traffic signals correctly during a simulation stage using different display systems. The designed test consists in to drive the motorcycle along a road and, at the same time, recognizing the kind of traffic signals proposed. To the recognition by the users, during the tests were proposed signs belonging to the vertical traffic signals. Although the simulation system created allows all the driving manoeuvres, the test was focused on assessing the user's attention to traffic signals along a straight path. This limitation was introduced after a series of tests that have demonstrated the inability to control the motorcycle model without a feedback system capable of providing appropriate stimuli to the user on the behaviour of the vehicle while driving. The results of these tests shows that the realization of a simulator for motorcycles, because of their instability, is more difficult than for cars.

So the study of the perception of vertical traffic signals by the user was realized in simple conditions of the road, without considering the presence of other vehicles and with only objects like trees or lamp-posts to obstruct the correct visibility of the signs. The decision of testing the system on a straight path permits to release the user by the problem of the control of the vehicle and to focus his/her attention only on the perception of the traffic signals.

For the tests were taken into account two subsets of the regulatory signs group as defined by the United Nations
Economic and Social Council in the Vienna Convention on Road Signs and Signals of 1968 [15]: the traffic danger signals and those of obligation. To annoy the visibility of the users during the tests, a set of other extra signals were added to the scenario, such as prohibition signals. The user was called to identify the various types of signals and, in addition, to find a specific symbol among those. The traffic signal specifically requested during the tests belongs to the subgroup of warning signs. The signal in question is that which indicates driving with caution due to the presence of children (Fig. 7).

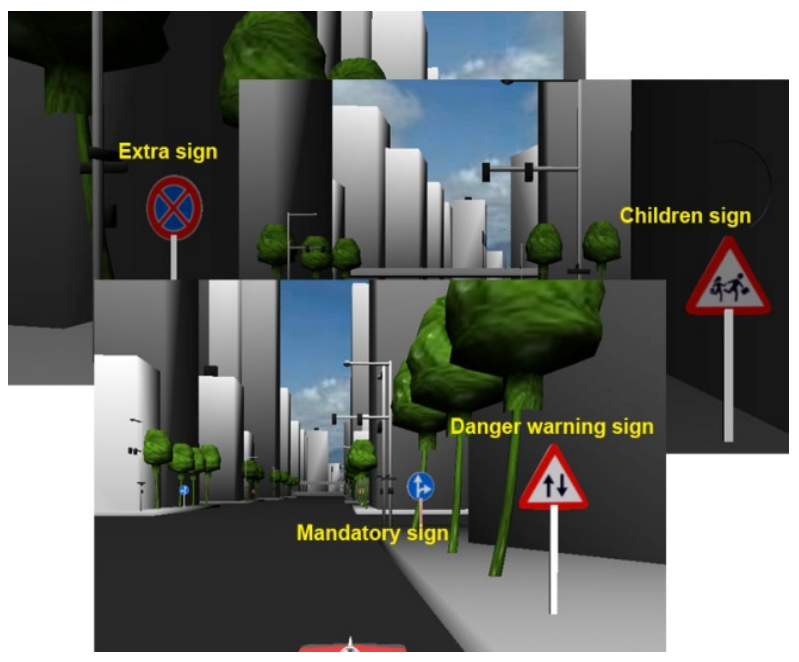

Fig. 7. Example of traffic signals added to the simulation scenario.

As stated above, the user interaction for an indication of the recognition of the traffic signal was achieved by means of a few buttons on the keyboard of the computer used for carrying out the tests.

It was established that the simulation begins when the user presses the accelerator button, corresponding to the "arrow up" key on the keyboard. Since that time the vehicle is in motion and its speed is governed by the same user but it cannot exceed the maximum speed prescribed for the simulation environment, equal to $50 \mathrm{~km} / \mathrm{h}$ and $90 \mathrm{~km} / \mathrm{h}$ respectively for the urban and rural environment.

To reduce the problems related to the possible instability of the vehicle at low speed or resulting from wrong maneuvers by the user, the control channel of the roll movement has been disabled. The motorcycle is so stable throughout the simulation path, which, as already above told, is straight.

When the user encounters one of the signals prescribed in the tests is called to press the button corresponding to that group of signals on keyboard. So, for mandatory signs the user had to press the $\mathrm{Z}$ button, while for the warning signs he/she had to press the $X$ button, and then, for the "Children" sign he/she had to press the $\mathrm{C}$ button on the keyboard. No keys should be pressed when they met the other types of signals. For each simulation were recorded the number and type of signal detected by the users and the time of the perception, starting from the beginning of the simulation. Fig. 8 show the flowchart of the simulation protocol implemented. 


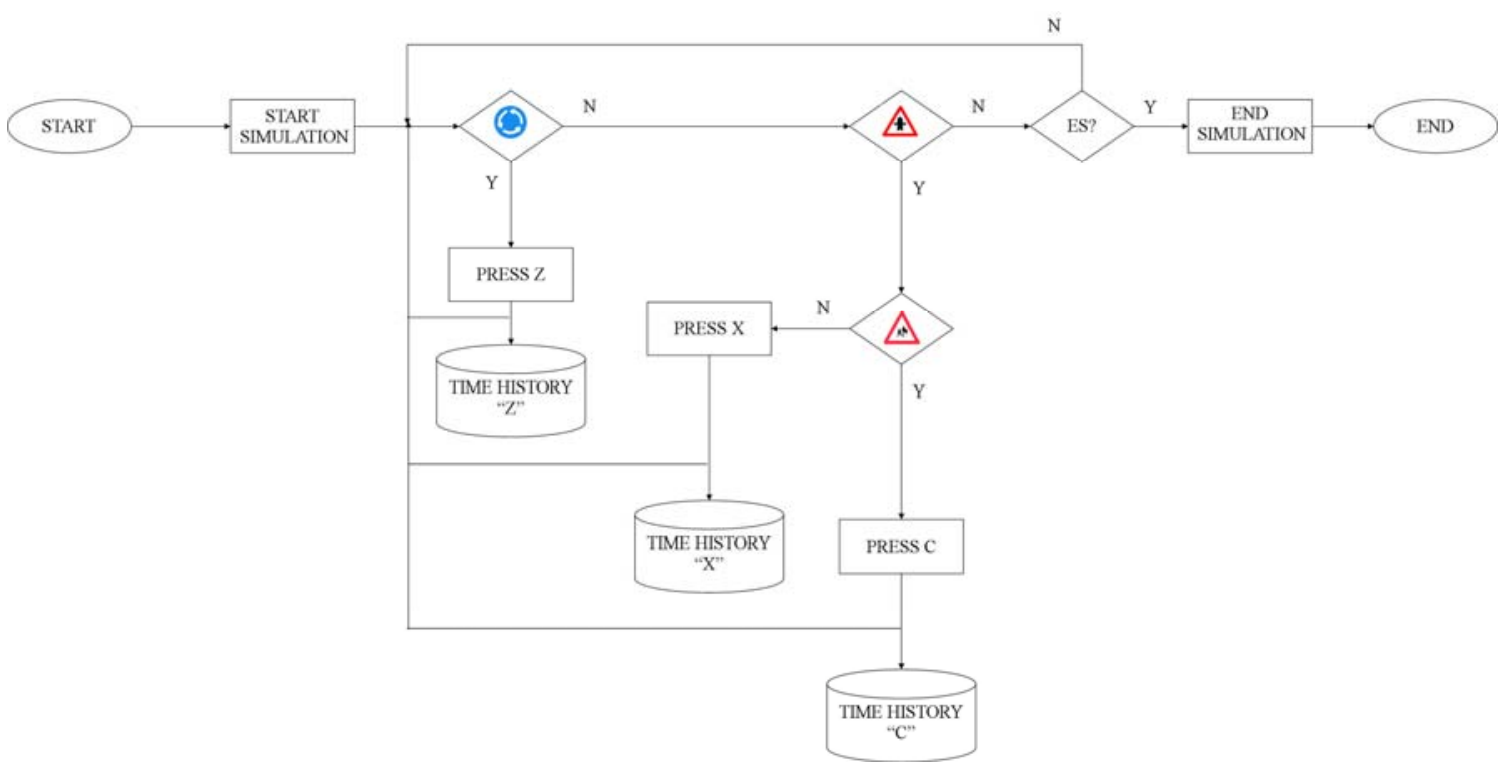

Fig. 8. Flowchart of the simulation protocol.

\section{RESULTS}

The test was conducted using 20 people between 16 and 68 years old. They were selected among people who had already acquired the driving license, to cars or motorcycles, from some years.

The test was preceded by a brief explanation of the simulation and the type of traffic signals that users had to identify.

The tests have been conducted in two different phases: the first one phase of the test has been conducted using a computer monitor as visualization system; in the second one phase the monitor was replaced by the HMD.

For each of the two phases any user has conducted the following six tests:

- Urban environment in dry conditions (sun);

- Rural environment in dry conditions (sun);

- Urban environment in wet conditions (rain);

- Rural environment in wet conditions (rain);

- Urban environment in low visibility conditions (fog);

- Rural environment in low visibility conditions (fog).

Tests were conducted trying to avoid the memorization by the users of the position of the traffic signals proposed. To do this, each simulations was conducted at a distance of time from each other and, from time to time, the position of the symbols of the road signs was changed. This last action was realized without change the total number of the signals in the test and their number for each membership group . This helped to prevent the memorization by the users of the position of the signals proposed in the two environments. Only the position of the "Children" sign did not change during the entire test.
The two environments realized are characterized by the presence of the following traffic signals:

- Urban environment:
o 2 Mandatory signs;
o 4 Warning signs;
o 1 "Children" sign;
o 8 Extra signs.

- Rural environment:
o 2 Mandatory signs;
o 7 Warning signs;
o 1 "Children" sign;
o 5 Extra signs.

In the following sections we report the results of the tests. For each test we report the results on the average number of the traffic signals recognized by the users according to the group, the number of users who have identified the "Children" sign and the average time for such recognition. The "Children" sign was also included between the warning signs in the statistic.

\subsection{Phase 1 - Urban environment}

Remembering that the viewing in the phase 1 was achieved by the use of a computer monitor, this is the case of the urban environment scenario.

Table 1 reports the statistical values of average and standard deviation of the traffic signals recognized for each test.

Concerning with the results obtained by the test, related to the perception of the "Children" sign, in figure 9 is reported the time from the start of the simulation to the recognition of the sign for each user. Finally, in table 2 is reported how many people detected the presence of the "Children" sign and the average time of their recognition, from the start of the 
simulation.

TABLE 1. TEST RESULTS OF THE PHASE 1 FOR THE URBAN ENVIRONMENT

\begin{tabular}{|l|c|c|c|c|}
\hline \multirow{2}{*}{} & \multicolumn{2}{|c|}{ Mandatory signs } & \multicolumn{2}{c|}{ Warning signs } \\
\cline { 2 - 5 } & Average & St. dev. & Average & St. dev. \\
\hline Sun & 2,90 & 1,71 & 6,70 & 1,99 \\
\hline Rain & 1,85 & 0,81 & 6,05 & 1,70 \\
\hline Fog & 1,80 & 0,77 & 5,35 & 1,81 \\
\hline
\end{tabular}

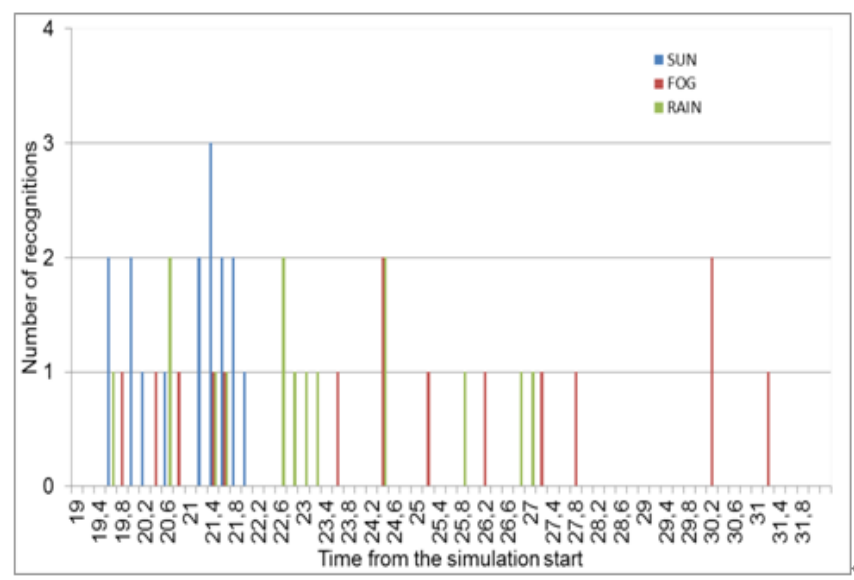

Fig. 9. Recognition time of "Children" sign from the start of the simulation during phase 1 - Urban environment.

TABLE 2. NUMBER OF RECOGNITIONS AND AVERAGE TIME FOR THE “CHILDREN" SIGN DURING PHASE 1 - URBAN ENVIRONMENT

\begin{tabular}{|l|c|c|}
\hline & Number of recognitions & Average time [s] \\
\hline Sun & 16 & 21,00 \\
\hline Rain & 15 & 23,10 \\
\hline Fog & 15 & 24,90 \\
\hline
\end{tabular}

\subsection{Phase 1 - Rural environment}

Such as for the previous step, Table 3 reports the statistical values of average and standard deviation of the traffic signals recognized.

In Fig. 10 are reported the results related to the perception of the "Children" sign and in table 4 is reported how many people have detected the presence of the "Children" sign and the average time of their recognition, from the start of the simulation in the rural environment case.

TABLE 3. TEST RESULTS OF THE PHASE 1 FOR THE RURAL ENVIRONMENT

\begin{tabular}{|l|c|c|c|c|}
\hline \multirow{2}{*}{} & \multicolumn{2}{|c|}{ Mandatory signs } & \multicolumn{2}{c|}{ Warning signs } \\
\cline { 2 - 5 } & Average & St. dev. & Average & St. dev. \\
\hline Sun & 2,75 & 1,59 & 6,42 & 1,96 \\
\hline Rain & 2,30 & 1,22 & 6,25 & 1,36 \\
\hline Fog & 3,15 & 1,95 & 6,91 & 1,29 \\
\hline
\end{tabular}

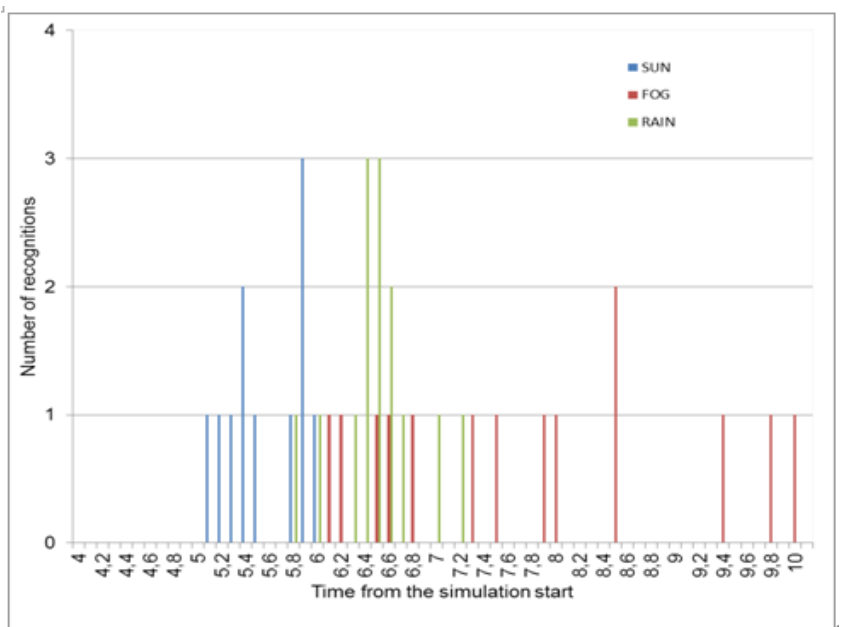

Fig. 10. Recognition time of "Children" sign from the start of the simulation during phase 1 - Rural environment.

TABLE 4. NUMBER OF RECOGNITIONS AND AVERAGE TIME FOR THE “CHILDREN” SIGN DURING PHASE 1 - RURAL ENVIRONMENT

\begin{tabular}{|l|c|c|}
\hline & Number of recognitions & Average time [s] \\
\hline Sun & 11 & 5,70 \\
\hline Rain & 14 & 6,50 \\
\hline Fog & 14 & 7,80 \\
\hline
\end{tabular}

\subsection{Phase 2 - Urban environment}

The viewing in the phase 2 was achieved by the use of a Head Mounted Display traced by a camera-detection system. In this way the user can rotate his/her head and, controlling the orientation of the point of view, obtaining so a better description of the virtual environment.

This solution required a short training period to accustom the users to the position of the keyboard keys used for the interface. Indeed, the HMD does not allow the user to view the external environment and, because the required interaction was considered simple, it is preferred not to add elements to the visual representation, such as buttons or hands, other than those present in the first phase.

Finally, Table 5 reports the statistical values of average and standard deviation of the traffic signals recognized in the urban environment case.

In Fig. 11 are reported the results related to the perception of the "Children" sign and in table 6 is reported how many people detected the presence of the "Children" sign and the average time of their recognition, from the start of the simulation.

TABLE 5. TEST RESULTS OF THE PHASE 2 FOR THE URBAN ENVIRONMENT

\begin{tabular}{|l|c|c|c|c|}
\hline \multirow{2}{*}{} & \multicolumn{2}{|c|}{ Mandatory signs } & \multicolumn{2}{c|}{ Warning signs } \\
\cline { 2 - 5 } & Average & St. dev. & Average & St. dev. \\
\hline Sun & 2,25 & 1,21 & 4,70 & 1,75 \\
\hline Rain & 2,10 & 1,12 & 4,95 & 1,82 \\
\hline Fog & 2,35 & 1,26 & 5,15 & 1,76 \\
\hline
\end{tabular}




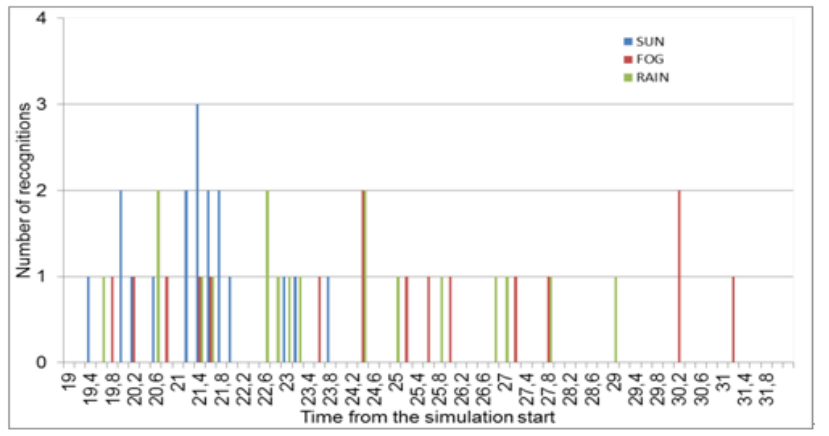

Fig. 11. Recognition time of "Children” sign from the start of the simulation during phase 2 - Urban environment.

TABLE 6. NUMBER OF RECOGNITIONS AND AVERAGE TIME FOR THE “CHILDREN” SIGN DURING PHASE 2 - URBAN ENVIRONMENT

\begin{tabular}{|l|c|c|}
\hline & Number of recognitions & Average time [s] \\
\hline Sun & 18 & 21,40 \\
\hline Rain & 18 & 23,80 \\
\hline Fog & 16 & 24,90 \\
\hline
\end{tabular}

\subsection{Phase 2 - Rural environment}

As with the previous steps, Table 7 reports the statistical values of average and standard deviation of the traffic signals recognized in the rural environment case.

In Fig. 12 are reported the results related to the perception of the "Children" sign and in table 8 is reported how many people detected the presence of the "Children" sign and the average time of their recognition, from the start of the simulation.

TABLE 7. TEST RESULTS OF THE PHASE 2 FOR THE RURAL ENVIRONMENT

\begin{tabular}{|l|c|c|c|c|}
\hline \multirow{2}{*}{} & \multicolumn{2}{|c|}{ Mandatory signs } & \multicolumn{2}{c|}{ Warning signs } \\
\cline { 2 - 5 } & Average & St. dev. & Average & St. dev. \\
\hline Sun & 2,60 & 1,50 & 6,55 & 1,76 \\
\hline Rain & 2,40 & 0,99 & 7,20 & 1,47 \\
\hline Fog & 2,50 & 1,64 & 6,95 & 1,36 \\
\hline
\end{tabular}

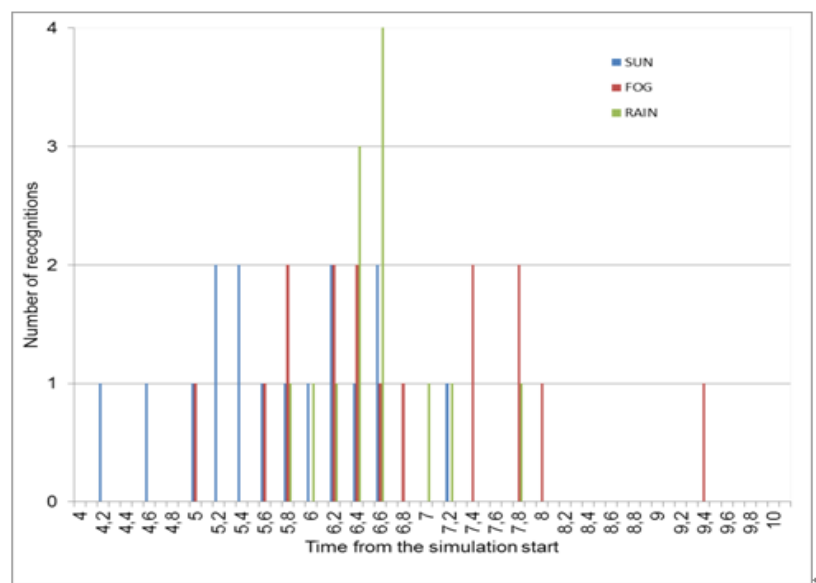

Fig. 12. Recognition time of "Children” sign from the start of the simulation during phase 2 - Rural environment.
TABLE 8. NUMBER OF RECOGNITIONS AND AVERAGE TIME FOR THE “CHILDREN” SIGN DURING PHASE 2 - RURAL ENVIRONMENT

\begin{tabular}{|l|c|c|}
\hline & Number of recognitions & Average time [s] \\
\hline Sun & 16 & 5,70 \\
\hline Rain & 15 & 6,60 \\
\hline Fog & 16 & 6,80 \\
\hline
\end{tabular}

\section{DISCUSSION}

Aiming the attention on the time required by the users to identify the warning "Children" sign, it is evident how this increases from the dry to the wet conditions, until to the worst situation of fog. The test underlines a greater difficulty of perception of this signs in an urban than in a rural environment. In fact the amplitude of the time interval in which the users indicated the presence of the sign is greater in the urban environment, approximately 15 seconds against the 8 seconds measured in the rural environment. This probably indicates that urban environment requires a greater attention to the motorcycle rider. Note that the variation in climatic conditions is matched by an extension in time used for the recognition of the traffic signals and, obviously, of the simulation. This is due to the reduction of vehicle speed realized by the users in the conditions of rain and fog.

The introduction of a traced HMD in the second phase of the work has improved the results of the simulation. Focusing on the tables II, IV, VI and VIII, it is evident how the possibility of moving the point of view in the virtual environment with rotations of the head permits to follow the crossing of the road signs, dedicating more time to the comprehension of the traffic signals. In fact, the warning "Children" sign was identified by a greater number of users during the phase 2 of the test, both in urban and in rural environment.

It is also possible to do a similar comparison for the number of mandatory and warning signs perceived in each phase of the test. In phase 2 the average that results from the tests of the various signs is closer to the real number of traffic signals positioned in the path. This is considered an effect of a better perception by the users.

In many cases, the number of road signs reported is sometimes higher than the number of signals proposed in the different groups. This is considered attributable to two factors: a lack of knowledge of road signs from users who participated in the test and by errors caused by a certain difficulty for the users to remember the association between sign and button to press

\section{CONCLUSIONS}

The reported results allow to understand the effectiveness of the visualization system created. Despite the small number of the test population, the results offer interesting perspective for the development of subsequently investigations. The introduction of a HMD improves the simulation experience and the capacity of perception of the traffic signals by the user. A new 
improvement, to the analysis of this perception capacity, could be obtained with the construction of a mock-up of the motorcycle, able to reproduce some dynamic situations of driving. In this case the user may adapt his posture to real position required by the driving of the motorcycle, obtaining diverse conditions of visibility. The authors believe that driving influences the perception of traffic signals and their understanding. In fact, tests conducted to constant speed of the vehicle and without engaging the user in the driving, as proposed in [14], led to better results in the perception of the traffic signals.

Some issues concerning the methodology of implementation and the purpose of the test are still open. If the vehicle dynamics is introduced in the simulation, it is considered necessary to change the mode of the traffic signal recognition. In fact, it is considered difficult to ask to the users to press a specific key, to identify the traffic signal, and maintain, simultaneously, an appropriate driving posture. So, it is necessary to study new forms of interaction for the communication of the perception of the signal by the user. One form is definitely to record the user's behaviour near to the signal, as proposed in several studies, but some traffic signals may not require specific actions. Another form of interaction could be obtained by means of the introduction of a vocal acquisition system. The latter solution could lend itself to interesting developments.

Thanks to the results from this study, especially when comparing those obtained in dry, rain and fog conditions, is believed that the use of an immersive virtual reality system for the training of the riders improves their skill and their sense of responsibility while driving. The introduction in the simulation scenario of more realistic traffic situations, such as the presence of other vehicles, pedestrians or animals, can provide a tool to help the drivers to overcome the complex cognitive demands that the traffic environment imposes.

\section{ACKNOWLEDGEMENTS}

The authors thank the participants of our study for their time and comments.

The study is part of the FIRB project (RBIP06AWF9) on "Highly innovative motorcycles with very low emission direct injection engines, active suspensions, assisted braking and new materials" financed by Italian MIUR.

\section{REFERENCES}

[1] European Commission, (2001), "White Book on transports European Policys for 2010: Time to Decide".

[2] http://ec.europa.eu/information_society/activities/esafety/index_en.htm European Commission web site on eSafety

[3] ERF European Union Road Federation, (2008), "European Road Statistic 2008”, http://www.erscharter.eu/resourcedocuments/9558.

[4] A. Borowsky, D. Shinar, T. Oron-Gilad, (2010). Age, skill, and hazard perception in driving. Accident Analysis and Prevention, 42(4), 1240-1249.

[5] K. Yoshimoto, T. Suetomi, (2008), "The History of Research and Development of Driving Simulators in Japan”, Journal of Mechanical Systems for Transportation and Logistics, 1(2), pp.159-169.
[6] G. Yamasky, K. Aoky, Y. Miyamaru, K. Ohnuma, (1998), “Development of a Motorcycle Training Simulator”, JSAE Review, Vol. 19, pp. 81-85.

[7] S. Chiyoda, K. Yoshimoto, D. Kawasaki, Y. Murakami, T. Sugimoto, "Development of a motorcycle simulator using parallel manipulator and head mounted display," in Driving Simulation Conference (DSC00), Paris, France, 2000

[8] D. Ferrazzin, F. Salsedo, M. Bergamasco, (1999), "The MORIS simulator" Robot and Human Interaction, 1999. RO-MAN '99. 8th IEEE International Workshop, pp.136-141.

[9] V. Cossalter, A. Doria, R. Lot, (2004), "Development and validation of a motorcycle riding simulator," in World Automotive Congress (FISITA2004), Barcelona, Spain, May 2004.

[10] S. Ichimi, Y. Miyamaru, K. Aoki, K. Suzuki, (2006), "Development of Riding Trainer for Safety Riding Education and Its Features", Proceedings. JSAE Annual Congress, Japan

[11] http://www.koalagames.it/koalaweb/pages/GuidatuPro/guidatupro_guida _sicura.asp - Koala Games S.r.l.

[12] R. Gray, D. M. Regan, (2005), "Perceptual Processes Used by Drivers During Overtaking in a Driving Simulator", Human Factors: The Journal of the Human Factors and Ergonomics Society Summer 2005 47: 394-417

[13] F. Ferrandez, (Ed), (1995). L'étude détaillée d'accidents orientée vers la sécurité primaire, méthodologie de recueil et de pré-analyse. Presses de l'Ecole Nationale des Ponts et Chaussées, Paris, France.

[14] S.T. Chrysler, J. Wright, A. Williams (2004). 3D Visualization as a Tool to Evaluate Sign Comprehension (Rep. No. SWUTC/04/167721-1). College Station, TX: Southwest University Transportation Center

[15] United Nations Economic and Social Council, (1968), Convention on road signs and signals (E/CONF.56/17/Rev.1/Amend.1), 8 November 1968, Vienna.

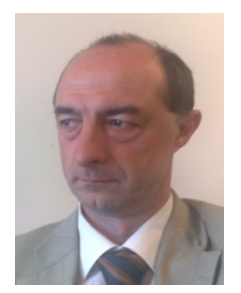

Roberto Vigano graduated in Mechanical Engineering at the Politecnico di Milano, then he achieved his Ph.D. in Applied Mechanics in the same University. $\mathrm{He}$ is Associate Professor in Methods and Tools for Industrial Design at the School of Industrial Engineering at the Politecnico di Milano. His research activity is mainly dedicated to the fields of Computer Aided Design, Virtual Simulation and Industrial Engineering Design Methods.

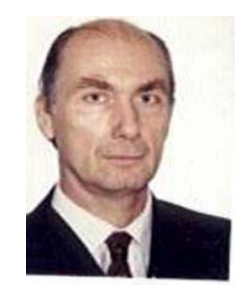

Edoardo Rovida graduated in Mechanical Engineering at the Politecnico di Milano.He is Full Professor in Methods and Tools for Industrial Design at the School of Industrial Engineering at the Politecnico di Milano. His research activity is mainly dedicated to the fields of Methodic Design, Technical Communication, Cultural Heritage in Mechanical Field.

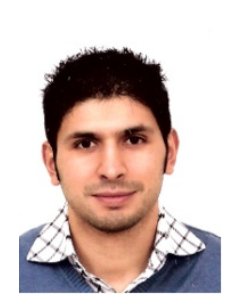

Riccardo Vincenti graduated in Mechanical Engineering at the Politecnico di Milano, he is currently studying for a Ph.D. in Mechanical Engineering in the same University. His research activity is dedicated to the Virtual Simulation in Engineering Design.

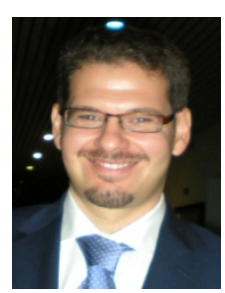

Marco Ramondino graduated in Mechanical Engineering at the Politecnico di Milano. He is research fellow at the Mechanical Department to the Politecnico di Milano. His research activity is dedicated to the Virtual Simulation in Engineering Design. 